\title{
Microwave Assisted Reaction of Condensed Thiophenes With Electron Poor Olefins
}

\author{
Khadijah M. Al-zaydi* and Mohamed H. Elnagdi ${ }^{\dagger}$ \\ Department of Chemistry, Girls College of Education, Jeddah, P.O.50918 Jeddah 21533, Kingdom of Saudi Arabia \\ "Department of Chemistr; Faculty of Science; Cairo University, Giza-A. R. Egypt \\ Repnint requested to Khadijah M. Al-Zaydi
}

(2003.5.27 접수)

\section{Microwave Assisted Reaction of Condensed Thiophenes With Electron Poor Olefins}

\author{
Khadijah M. Al-zaydi* and Mohamed H. Elnagdi ${ }^{\dagger}$ \\ Department of Chemistry, Girls College of Education, Jeddah. P.O.50918 Jeddah 21533, Kingdom of Saudi Arabia \\ ${ }^{4}$ Department of Chemistry; Fuculty of Science; Cairo University, Giza-A. R. Egypt \\ Reprint requested io Khadijah $M$. Al-Zaydi \\ (Rcceived May 27, 2003)
}

요 약. 아미노싸이에노쾨리다진(1a, b)과 하미노싸이에노쿠마린(2)은 DMFDMA와 축합반웅을 하여 아미딘(3a, b) 을 형성한다. 이 화합물들을 $\mathrm{N}$-페널말레이마이드와 반응시키면 화합물 9와 10이 언어진다. 반면에 3a, b, 4, 18, 19, 20을 말레산 무수물과 반웅시키면 포밀 유도체인 $5 \mathrm{a}, \mathrm{b}, 6,21,22,23$ 든이 얻어진다. 아미딘 화합물 3a, b 를 다이 에털 퓨마레이트와 반응시키면 가수분해산물인 아미던 14를 거쳐 11 이 얻어진다. N-페널말레이마이드를 마이크로웨이브 요븐에서 반응시키면 $[2+2]$ 와 [2+2+2] 고리첨가반옹 산눌이 얻어진다.

주제어: 마이크로웨히브, 싸이오펜, 고리첨가반응

\begin{abstract}
Aminothienopyridazines 1a. b and aminothienocoumarin 2 condensed with DMFDMA to yield amidines $3 \mathbf{a}, \mathrm{b}$ and 4 . These compounds reacted with $\mathrm{N}$-phenylmaleimide to yield 9 and 10 . On the other hand reacting $3 a, b, 4,18,19$ and 20 with maleic anhydride afforded only the formylated derivatives $5 a, b, 6,21,22$ and 23 respectively. The reaction of $\mathbf{3 a}$, $\mathbf{b}$ with diethyl fumarate afforded $\mathbf{1 1}$, formed most likely via hydrolysis of the amidine 14 during working up the reaction mixture. Irradiation of $N$-phenylnaleimide in microwave oven afforded $[2+2]$ and $[2+2+2]$ cycloaddition product.
\end{abstract}

Keywords: Microwave, Thiophene, Cycloaddition Reaction

\section{INTRODUCTION}

The cycloaddition of condensed aminothiophenes with electron poor olefins has proved to be an efficient route to benzo-fused heteroaromatics. ${ }^{1-5}$ It was suggested that presence of free amino groups is exsential for reactivity of condensed aminothiophenes as dienes in the Diels-Alder reaction. It occurred to us to see if substituted condensed aminothiophenes could also act as active dienes in the Diels-Alder reaction if drastic reaction condition were used.

\section{RESULTS AND DISCUSSION}

Consequenty we condensed amino thienopyridazines 1a, b and aminothienocoumarine 2 with dimethyl- 
formamide dimethylacetal (DMFDMA) and investigated the reactivity of the resulting amidines $\mathbf{3 a}, \mathbf{b}$ and 4 toward electron-poor olefins. In polar solvents, only hydrolysis of the amidine moiety in 3 and 4 afforded 5 and 6, respectively. Consequently we investigated the solventless addition of electronpoor olefins under a microwave irradiation, a technology that has been extensively utilized to affect Diels-Alder additions. ${ }^{6.7}$ N-Phenylmaleimide under this condition reacted smoothly with $3 a, b$ and 4 affording 9 and 10, which are assumed to be formed via intermediacy of cycloadducts 7,8 which then loses formamidine to yield the final products 9 , 10 (Scheme 1, 2).

In contrast to this, the reaction of $\mathbf{3}$ with diethyl fumarate in microwave oven has afforded phthalazine derivatives 11 formed via initial formation of cycloadduct when treated with diethyl fumarate 12 then eliminate $\mathrm{H}_{2} \mathrm{~S}$ to yield the non-isolable intermediate 14 which is then hydrolysed to afford the compound 11. Compounds 14, 18, 19 and 20 were recovered unreacted when treated with diethyl fumarate under the similar condition. Under a variety of conditions, maleic anhydride failed to react with $3 a, b, 4,18,19$ and 20 . Onty hydrolysis products to the formyl derivatives $5,6,21,22$ and 23

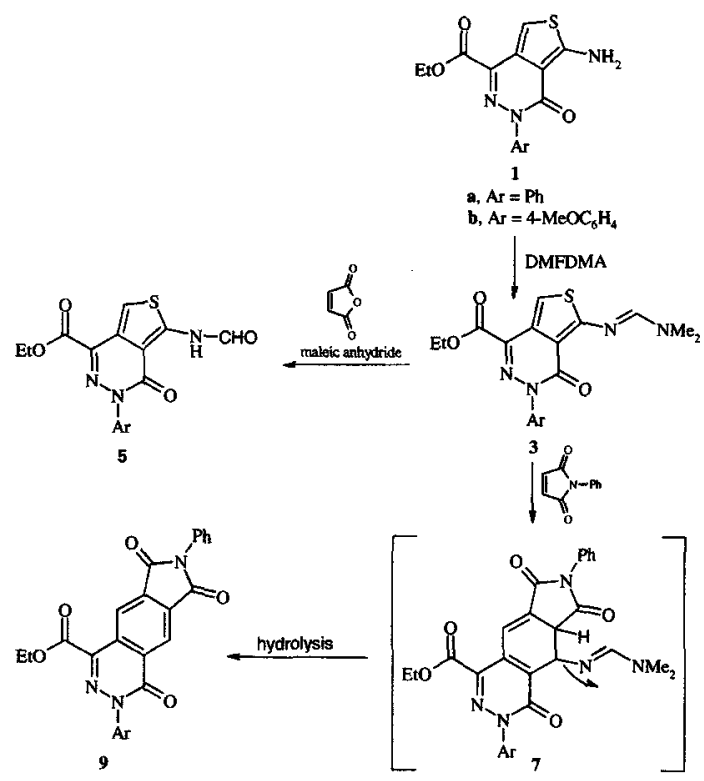

Scheme 1.

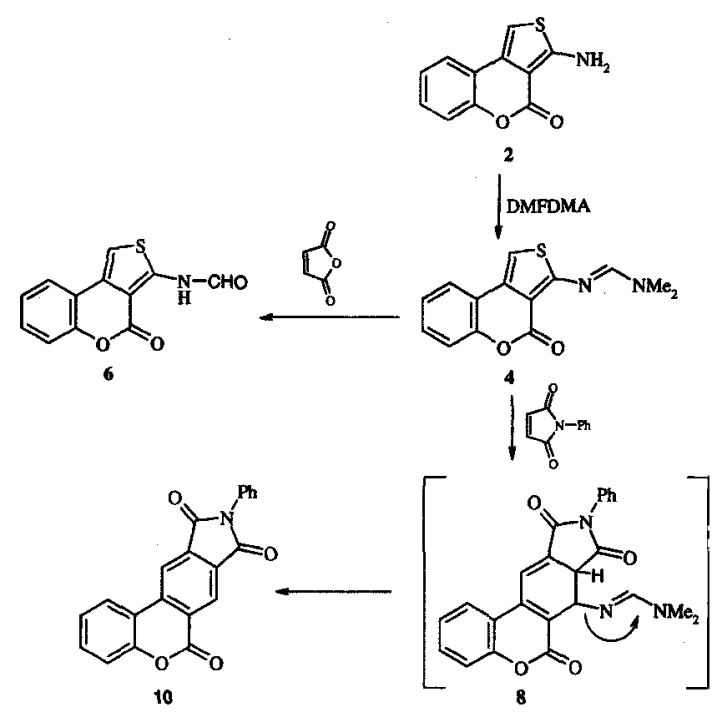

Scheme 2.
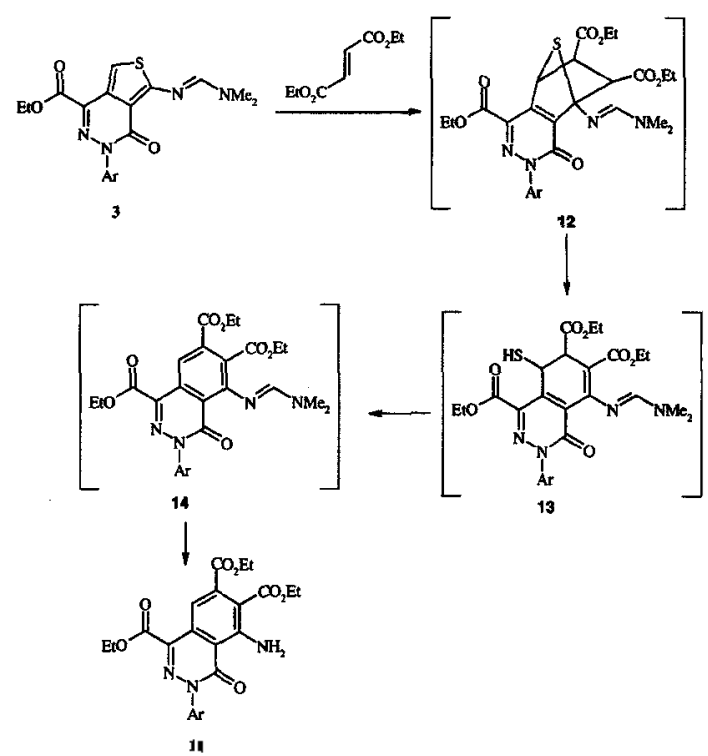

Scheme 3.

were observed (Scheme 3,4). The difference in the behavior of 3 toward maleic anthydride and $\mathrm{N}$-phenylmaleimide reflects their different activity as dienes in cycloaddition reactions. Consequently only hydrolysis occurs with maleic anhydride.

Formation of 18,19 and 20 has previously been reported via condensing 15,16 and 17 with dimethylfomamide-dimethylacetal (DMF DMA). ${ }^{8}$ So treatment of 18. 19 and 20 with maleic anhydride afforded 21,22 and 23 respectively. 


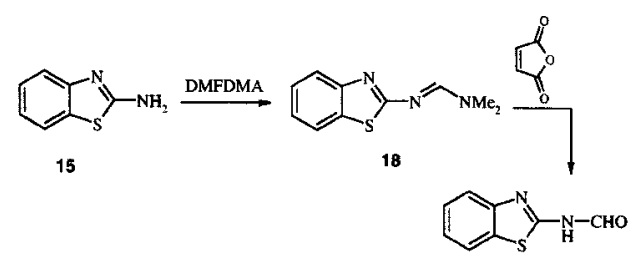

21

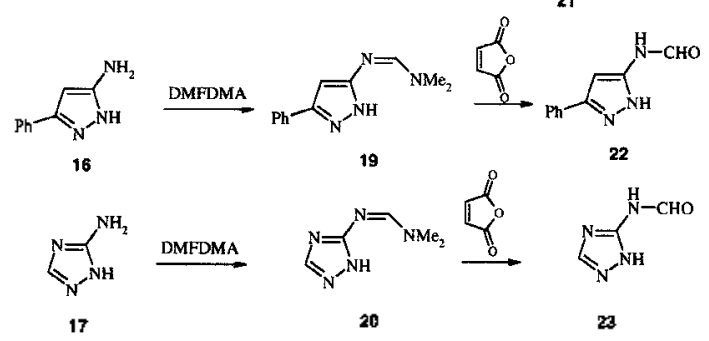

Scheme 4.

It is of value to report here that products of reacting aminothienocoumarine with $N$-phenylmaleimide were always contaminated with minor quantity of other products of MS $=346$ and 519 . These were the only isolable products from the reaction of $\mathbf{1 8}$, 19 and 20 with $N$-phenylmalimide. The same results were also obtained on irradiating $N$-phenylmaleimide in microwave oven. It is assumed to be the products of, most likely, nonconcerted $[2+2]$ dimerization of two molecules of $\mathrm{N}$-phenylmaleimide and the concerted $[2+2+2]$ trimerisation of three molecules of $\mathrm{N}$-phenylmalimide and thus assigned structures $\mathbf{2 4}$ and 25 respectively. To our knowledge, it is the first reported dimerization and trimerization under $N$ phenylmaleimide in microwave irradiation (Scheme 5). It seems that this reaction proceeds much faster than cycloaddition to the diene system in 18, 19 and 20. So this is reason why cycloaddition reaction did not proceed with these compounds.

\section{Experimental}

All melting points were measured on Galkenkamp electrothermal melting point apparatus and are uncorrected. IR spectra were recorded as $\mathrm{KBr}$ pellets on a Pye Unicam SP 3-300 Spectrophotometer. 'H NMR spectra were recorded in deuterated dimethylsulfoxide $\left(\mathrm{DMSO}-\mathrm{d}_{6}\right)$ or deuterated chloroform $\left(\mathrm{CDCl}_{3}\right)$ on either Varian Gemini ('H NMR at $200 \mathrm{MH} 7)$ or Bruker DPX ( ${ }^{1} \mathrm{H},{ }^{13} \mathrm{C}$ NMR at 400 $\mathrm{MHz}$ ) spectrometer using tetramethylsilane (TMS) as an internal reference and results are expressed as<smiles>CC1=C(CC2=C(C)C(=O)N(c3ccccc3)C2=O)C(=O)N(c2ccccc2)C1=O</smiles><smiles>CPN1C(=O)C2C(C1=O)C1C(=O)N(c3ccccc3)C(=O)C21</smiles><smiles></smiles><smiles>O=C1C2C3C(=O)N(c4ccccc4)C(=O)C3C3C4C(=O)N(c5ccccc5)C(=O)C4C4C(=O)N(c5ccccc5)C(=O)C4C3C2C(=O)N1c1ccccc1</smiles>

Schene 5 .

$\delta$ values. Mass spectra were performed on a Shimadzu GCMS-QP 1000 Ex mass spectrometer at $70 \mathrm{eV}$. Microwave irradiation was carried out using commercial microwave oven and irradiation power of $450 \mathrm{~W}$. Elemental analyses were carried out at the Microanalytical center of Cairo University.

General Procedure for the preparation of $3 a, b$,

\section{$4,18,19$ and 20}

Method A. $N, N$-Dimethylformamide dimethylacetal $(0.12 \mathrm{~mol})$ was added to each of $(\mathbf{1 a}, \mathbf{b}, \mathbf{2}$, $15,16,17)(0.1 \mathrm{~mol})$ in xylene and the reaction mixture was refluxed for 6 hours. The removal of solvent under reduced pressure yielded the crude proxluct which was crystallized from ethanol."

Method B. Compound (1a. b, 2, 15, 16, 17) (0.1 mol) and dimethylformamide dimethylacetal $(0.1$ mol) was placed in the microwave oven and irradiated at full power for 5-7 mins then left to cool to room temperalure, and the solid was collected and crystallized from ethanol.

5-(Dimethylaminomethylenamino)-4-oxo-3-phenyl-3,4-dihydro-thieno $[3,4-d]$ pyridazine-1-carboxylic acid ethyl ester $3 a$

Compound 3a was obtained as green crystals (method A: 61\%, method B: 90\%) mp $153^{\circ} \mathrm{C}$. Analysis for $\mathrm{C}_{18} \mathrm{H}_{13} \mathrm{~N}_{4} \mathrm{O}_{3} \mathrm{~S}(370.43)$. Calcd: $\mathrm{C}, 58.36 ; \mathrm{H}_{4}$ $4.90 ; \mathrm{N}, 15.12 \%$ Found: C, $58.39 ; \mathrm{H}, 4.91 ; \mathrm{N}, 15.14 \%$. IR $\left(\mathrm{v} / \mathrm{cm}^{-1}\right)$ : 1729 (CO ester) 1711 (ring CO), ${ }^{1} \mathrm{H} \mathrm{NMR}$ $(\delta \mathrm{ppm}): 1.38\left(\mathrm{t}, 3 \mathrm{H}, \mathrm{CH}_{2} \mathrm{CH}_{2}\right) ; 3.07\left(\mathrm{~s}, 3 \mathrm{H}, \mathrm{CH}_{3}-\mathrm{N}\right)$ : $3.10\left(\mathrm{~s}, 3 \mathrm{H}, \mathrm{CH}_{3}-\mathrm{N}\right) ; 4.42\left(\mathrm{q}, 2 \mathrm{H}, \mathrm{CH}_{2}\right) ; 702-7.60(\mathrm{~m}$, $5 \mathrm{H}, \mathrm{Ar}-\mathrm{H}) ; 7.27(\mathrm{~s}, \mathrm{IH}$, thienyl $\mathrm{H}) ; 7.89 \mathrm{ppm}(\mathrm{s}, 1 \mathrm{H}$. amidine $\mathrm{H})$. ms: $\mathrm{M}^{-}(370)$. 
5-(Dimethylaminomethylenamino)-3-(4-methoxypheny)-4-oxo-3,4-ithydro-thieno[3, 4d]pyridazine1-carboxylic acid ethyl ester $\mathbf{3 b}$

Compound 3b was obtained as yellow crystals (method A: 62\%, method B: $92 \%$ ) mp $147^{\circ} \mathrm{C}$. Analysis for $\mathrm{C}_{39} \mathrm{H}_{20} \mathrm{~N}_{4} \mathrm{O}_{4} \mathrm{~S}$ (400.45): Calcd: C, 56.99; $\mathrm{H}, 5.03 ; \mathrm{N}, 13.99$. Found C, 56.97; H, 5.11; N, $14.01 \%$.

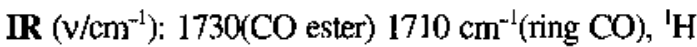
NMR $(\delta \mathrm{ppm}): 1.39\left(\mathrm{t}, 3 \mathrm{H}, \mathrm{CH}_{3} \mathrm{CH}_{2}\right) ; 3.08(\mathrm{~s}, 3 \mathrm{H}$, $\left.\mathrm{CH}_{3}-\mathrm{N}\right) ; 3.10\left(\mathrm{~s}, 3 \mathrm{H}, \mathrm{CH}_{3}-\mathrm{N}\right) ; 3.85\left(\mathrm{~s}, 3 \mathrm{H}, \mathrm{OCH}_{3}\right)$; 4.42(q. $\left.2 \mathrm{H}, \mathrm{CH}_{2}\right) ; 6.93-7.00(\mathrm{~m}, 2 \mathrm{H}$, Aryl $\mathrm{H}-3,5)$; 7.28(s, $1 \mathrm{H}$, thienyl $\mathrm{H}) ; 7.4-7.5(\mathrm{~m}, 2 \mathrm{H}$, Aryl $\mathrm{H}-2, \mathrm{H}-$ 6); $7.88 \mathrm{ppm}(\mathrm{s}, \mathrm{lH}$, amidine $\mathrm{H}) .{ }^{13} \mathrm{C} \mathrm{NMR}(\delta \mathrm{ppm})$ : $14.37\left(\mathrm{CH}_{3} \mathrm{CH}_{2}\right) ; 34.97\left(\mathrm{CH}_{3}-\mathrm{N}\right) ; 40.84\left(\mathrm{CH}_{3}-\mathrm{N}\right)$; $55.60\left(\mathrm{OCH}_{3}\right) ; 61.91\left(\mathrm{OCH}_{2}\right) ; 112.29,114.03,114.18$, $127.84,128.80,133.08,134,53,156.51$, (aromatic and heterocyclic carbon) 157.97 (amidine $\mathrm{CH}$ ) and $158.91,163.45$ (2CO). ms: $\mathrm{M}^{+}(400)$.

3-N,N-Dimethylaminothieno[3,4:3, $\left.4^{\prime}\right]$ benzo $[b]$ pyran-4-one 4

Compound 4 was obtained as gren crystals (method A: $72 \%$, method B: $93 \%$ ) mp $148^{\circ} \mathrm{C}$. Analysis for $\mathrm{C}_{14} \mathrm{H}_{12} \mathrm{~N}_{2} \mathrm{O}_{2} \mathrm{~S}$ (272.32): Calcd: C, 61.75; H, 4.44; N, 10.29. Found: C, $61.77 ; \mathrm{H}, 4.45 ; \mathrm{N}, 10.30 \%$. IR (v/ $\mathrm{cm}^{-1}$ ): $1690 \mathrm{~cm}^{-1}$ (ring CO), ${ }^{1} \mathrm{H}$ NMR ( $\delta \mathrm{ppm}$ ): 2.47 $\left(5,3 \mathrm{H}, \mathrm{CH}_{3}\right) ; 2.51\left(\mathrm{~s}, 3 \mathrm{H}, \mathrm{CH}_{3}\right) ; 7.13-7.7 \mathrm{ppm}(\mathrm{m}$, $6 \mathrm{H}, \mathrm{Ar}-\mathrm{H}$, thienyl $\mathrm{H}$ and amidine $\mathrm{H})$. $\mathrm{ms}: \mathrm{M}^{+}(272)$.

$N$-Benzothiazol-2-yl-N,N-dimethylformamidine 18

Compound 18 was obtained as colourless crystals (75\%) mp $111^{\circ} \mathrm{C}$. Analysis for $\mathrm{C}_{10} \mathrm{H}_{11} \mathrm{~N}_{3} \mathrm{~S}(205.28)$; Calcd: C, 58.51; H, 5.40; N, 20.47. Found: C, $58.57 ; \mathrm{H}, 5.45 ; \mathrm{N}, 20.30 \%$. ' $\mathrm{H}$ NMR $(\delta \mathrm{ppm})$ : $3.02\left(\mathrm{~s}, 3 \mathrm{H}, \mathrm{CH}_{3}-\mathrm{N}\right) ; 3.04\left(\mathrm{~s}, 3 \mathrm{H}, \mathrm{CH}_{3}-\mathrm{N}\right) ; 7.13-7.65$ (m, $4 \mathrm{H}, \mathrm{Ar}-\mathrm{H}$ ); $8.28 \mathrm{ppm}\left(\mathrm{s}, 1 \mathrm{H}\right.$, amidine $\mathrm{H}$ ). ${ }^{13} \mathrm{C}$ NMR ( $\delta$ ppm): $35.12\left(\mathrm{CH}_{3}-\mathrm{N}\right) ; 40.97\left(\mathrm{CH}_{3}-\mathrm{N}\right) ; 120.58$, $121.23,122.85,125.74,133.33,152.11$ (phenyl carbon), 156.54 (thiazole C-2) and 173.61 (amidine). ms: $\mathrm{M}^{+}(205)$.

$N, N$-Dimethyl- $N$-(5-phenyl-2 $H$-pyrazol-3-yl) formamidine 19

Compound 19 was obtained as buff crystals (method A: $78 \%$, method B: $89 \%$ ) mp $175^{\circ} \mathrm{C}$. Analysis for $\mathrm{C}_{12} \mathrm{H}_{14} \mathrm{~N}_{4}(214.27)$ : Calcd: C, 67.27; H, 6.59; N, 26.15. Found: C, 67.31; H, 6.60; N, 26.17\%. IR (v/ $\left.\mathrm{cm}^{-1}\right): 3200(\mathrm{NH})$ and $3074 \mathrm{~cm}^{-1}(\mathrm{CH}),{ }^{1} \mathrm{H}$ NMR $(\delta$ ppm): $2.97\left(\mathrm{~s}, 3 \mathrm{H}, \mathrm{CH}_{3}-\mathrm{N}\right) ; 3.04\left(\mathrm{~s}, 3 \mathrm{H}, \mathrm{CH}_{3}-\mathrm{N}\right) ; 6.13$ (s, 1H, pyrazol H-4); 7.34-7.78 (m, 5H, Ar-H); 7.86 (s, 1H, amidine $\mathrm{CH}$ ) and $11.9 \mathrm{ppm}(\mathrm{br}, \mathrm{lH}, \mathrm{NH}$ ). ${ }^{13} \mathrm{C}$ NMR (6 ppm): $34.94\left(\mathrm{CH}_{3}-\mathrm{N}\right) ; 40.66\left(\mathrm{CH}_{3}-\mathrm{N}\right)$; 87.83 (pyrazolyl C-4); 125.48, 127.50, 128.60, 133.77 (phenyl carbon), 150.0 (pyrazolyl C-2); 153.0 (pyrazolyl $\mathrm{C}-5$ ) and 155.27 (amidine $\mathrm{CH}$ ). ms: $\mathrm{M}^{+}(214)$.

$N, N$-Dimethyl- $N$ - $(2 H-[1,2,4]$ triazol-3-yl)formamidine 20

Compound 20 was obtained as colourless crystals (method A: $76 \%$, method B: $85 \%$ ) mp $97{ }^{\circ} \mathrm{C}$. Analysis for $\mathrm{C}_{5} \mathrm{H}_{4} \mathrm{~N}_{5}$ (139.16): Calcd: $\mathrm{C}, 43.15 ; \mathrm{H}$, $6.52 ; \mathrm{N}, 50.33$. Found: C, 43.20; H, 6.51; N, 50.36\%. IR $\left(\mathrm{v} / \mathrm{cm}^{-1}\right): 3325(\mathrm{NH}), 2980 \mathrm{~cm}^{-1}(\mathrm{CH}),{ }^{\prime} \mathrm{H}$ NMR $(\delta \mathrm{ppm}): 2.96\left(\mathrm{~s}, 3 \mathrm{H}, \mathrm{CH}_{3}-\mathrm{N}\right) ; 3.04\left(\mathrm{~s}, 3 \mathrm{H}, \mathrm{CH}_{3}-\mathrm{N}\right)$; 7.96(s, $1 \mathrm{H}$, amidine $\mathrm{CH}) ; 8.3(\mathrm{~s}, 1 \mathrm{H}$, trizol H-3); and $12.7 \mathrm{ppm}$ (br, $1 \mathrm{H}, \mathrm{NH}$ ). ms: $\mathrm{M}^{+}$(139).

Reaction of $3 \mathrm{a}, 3 \mathrm{~b}, 4,18,19$ and 20 with maleic anhydride

\section{General Procedure.}

Method A. A mixture of each of $(3 a, b, 4,18$, $19,20)(0.1 \mathrm{~mol})$ and maleic anhydride $(0.1 \mathrm{~mol})$ was heated at $200^{\circ} \mathrm{C}$ for $30-60$ mins, left to cool and triturated with ethanol. The solid product, so formed, was collected by filtration and crystallized from ethanol.

Method B. A mixture of each of (3a, b, 4, 18, 19 , 20) $(0.1 \mathrm{~mol})$ and maleic anhydride $(0.1 \mathrm{~mol})$ was placed in the microwave oven and irradiated at 450 W for 2-5 mins, then left to cool to room temperature, and the solid was collected and crystallized from ethanol.

5-Formylamino-4-oxo-3-phenyl-3,4-dihydrothieno $[3,4-d]$ pyridazine-1-carboxylic acid ethyl ester 5a

Compound 5a was obtained as green crystals (method A: 53\%, method B, 2 min : 65\%) mp 249$250^{\circ} \mathrm{C}$. Analysis for $\mathrm{C}_{16} \mathrm{H}_{13} \mathrm{~N}_{3} \mathrm{O}_{4} \mathrm{~S}$ (343.36): Calcd: C, $55.97 ; \mathrm{H}, 3.82 ; \mathrm{N}, 12.24$. Found: C, 55.99; H, 3.91: N, 12.14\%. IR ( $\left./ \mathrm{cm}^{-1}\right): 3345(\mathrm{NH}), 1731(\mathrm{CO}$ ester) $1690\left(\mathrm{CO}\right.$ ) and $1645 \mathrm{~cm}^{-1}$ (ring $\mathrm{CO}$ ), 'H NMR $(\delta \mathrm{ppm}): 1.43\left(\mathrm{~L}, 3 \mathrm{H}, \mathrm{CH}_{3}\right) ; 4.48\left(\mathrm{q}, 2 \mathrm{H}, \mathrm{CH}_{2}\right) ; 7.39$ $7.60(\mathrm{~m}, 5 \mathrm{H}, \mathrm{Ar}-\mathrm{H}) ; 7.96(\mathrm{~s}, 1 \mathrm{H}$, thienyl H); 8.55 (s, $1 \mathrm{H}, \mathrm{CHO}) ; 11.09 \mathrm{ppm}(\mathrm{s}, \mathrm{IH}, \mathrm{NH}) .{ }^{13} \mathrm{C}$ NMR $(\delta$ ppm): $14.37\left(\mathrm{CH}_{3} \mathrm{CH}_{2}\right) ; 62.38\left(\mathrm{OCH}_{2}\right) ; 112.88,115.71$, $125.38,126.03,128.34,128.99,133,96$ (aromatic 
and heterocyclic carbon) and 157.50, 158.95, 162.79 (3CO). ms: $\mathrm{M}^{+}$(343).

5-Formylamino-3-(4-methoxyphenyi)-4-oxo-3,4dihydrothieno $[3,4-d]$ pyrida-zine-1-carboxylic acid ethyl ester $5 b$

Compound $\mathbf{5 b}$ was obtained as yeilow crystals (method A: 30\%, method B, 2 min: 55\%) mp 228$230{ }^{\circ} \mathrm{C}$. Analysis for $\mathrm{C}_{17} \mathrm{H}_{15} \mathrm{~N}_{3} \mathrm{O}_{5} \mathrm{~S}$ (373.38): Calcd: C, 54.68; H, 4.05; N, 11.25. Found: C, 54.67; H. 4.11: N, $11.28 \%$. IR $\left(\mathrm{v} / \mathrm{cm}^{1}\right) ; 3340(\mathrm{NH}), 1731(\mathrm{CO}$ ester) $1690\left(\mathrm{CO}\right.$ ) and $1678 \mathrm{~cm}^{-1}$ (ring $\mathrm{CO}$ ), ${ }^{1} \mathrm{H}$ NMR ( $\delta \mathrm{ppm}): 1.39\left(\mathrm{t}, 3 \mathrm{H}, \mathrm{CH}_{3} \mathrm{CH}_{2}\right) ; 3.86\left(\mathrm{~s}, 3 \mathrm{H}, \mathrm{OCH}_{3}\right)$; 4.43(q, $\left.2 \mathrm{H}, \mathrm{CH}_{2}\right) ; 7.11$ (m. 2H, Aryl H-3, 5); 7.49 (m, 2H, Aryl H-2, H-6), 8.02(s. IH, thienyl H); 8.67(s, JH, CHO): 11.72ppm (s, 1H, NH). ms: $\mathrm{M}^{+}(373)$.

3-Formylaminothieno $[3,4: 3,4]$ benzo[ $b]$ pyran4-one 6

Compound 6 was obtained as pale green crystals (method A: 52\%, method B, 3 min: $89 \%$ ) mp $227-$ $228{ }^{\circ} \mathrm{C}$. Analysis for $\mathrm{C}_{1}, \mathrm{H}_{7} \mathrm{NO}_{3} \mathrm{~S}(245.25)$ : Calcd: C, 58.77; H, 2.88; N. 5.71. Found: C, 58.79; H. 2.91; $\mathrm{N}, 5.74 \%$. IR $\left(\mathrm{v} / \mathrm{cm}^{-1}\right): 3345(\mathrm{NH}), 1693(\mathrm{CO})$ and $1679 \mathrm{~cm}^{-1}$ (ring CO), ${ }^{1} \mathrm{H}$ NMR ( $\delta$ ppm); $6.95-7.52$ (m, 5H, Ar-H); 8.38 (s, 1H, CHO); $11.01 \mathrm{ppm}(\mathrm{s}$, $1 \mathrm{H}, \mathrm{NH})$. ms: $\mathrm{M}^{+}(245)$.

\section{$\mathrm{N}$-Benzothizol-2-ylformamide 21}

Compound 21 was obtained as green crystals (method A: $30 \%$, method B, 3 min: $62 \%$ ) mp $165^{\circ} \mathrm{C}$. Analysis for $\mathrm{C}_{\mathrm{B}} \mathrm{H}_{6} \mathrm{~N}_{2} \mathrm{OS}$ (178.21): Calcd: C, 53.92; H, 3.39; N, 15.72. Found: C, 53.97; H, 3.40; N, 15.80\%. ${ }^{1} \mathrm{H}$ NMR $(\delta \mathrm{ppm}): 7.14-7.68(\mathrm{~m}, 4 \mathrm{H}$, Ar$\mathrm{H}) ; 8.58(\mathrm{~s}, 1 \mathrm{H}, \mathrm{CHO})$ and $11.12 \mathrm{ppm}(\mathrm{s}, \mathrm{IH}, \mathrm{NH})$. ms: $\mathrm{M}^{+}(178)$.

\section{$\boldsymbol{N}$-(5-Phenyl-2H-pyrazol-3-yl)formamide $\mathbf{2 2}$}

Compound 22 was obtained as beige crystals (method A: 14\%, method B, 5 min: $35 \%$ ) inp 224$226^{\circ} \mathrm{C}$. Analysis for $\mathrm{C}_{10} \mathrm{H}_{9} \mathrm{~N}_{3} \mathrm{O}$ (187.20): Calcd: $\mathrm{C}$, 64.16; H, 4.85; N, 22.45. Found: C, 64.21; H, 4.89;

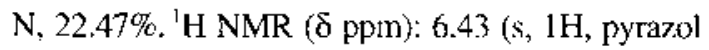
$\mathrm{H}-4) ; 7.34-7.79(\mathrm{~m}, 5 \mathrm{H}, \mathrm{Ar}-\mathrm{H}) ; 8.21$ (s, $1 \mathrm{H}, \mathrm{CHO})$ $11.01(\mathrm{ls}, 1 \mathrm{H}, \mathrm{NH})$ and $12.01 \mathrm{ppm}(1 \mathrm{~s}, 1 \mathrm{H}, \mathrm{NH})$. $\mathrm{ms}: \mathrm{M}^{+}(2 \mid 4)$.

\section{$\mathrm{N}$-(2H-[1, 2, 4]Triazol-3-yl)formamide 23}

Compound 23 was obtained as beige crystals (method A: $25 \%$, method B, 5 min: $45 \%$ ) mp $254^{\circ} \mathrm{C}$.
Analysis for $\mathrm{C}_{4} \mathrm{H}_{6} \mathrm{~N}_{4} \mathrm{O}(126.12)$; Calcd.: $\mathrm{C}, 38.09 ; \mathrm{H}$, 4.80; N, 44.42. Found: C, 38.10; H, 4.81; N, 44.46\%. ${ }^{1} \mathrm{H}$ NMR $(\delta \mathrm{ppm}): 8.3(\mathrm{~s}, 1 \mathrm{H}$, trizol H-3); $9.72(\mathrm{~s}$, 1H. CHO); 10.09 (1s, 1H, NH) and $12.7 \mathrm{ppm}$ (Is, $1 \mathrm{H}, \mathrm{NH})$. ms: $\mathrm{M}^{+}$(139).

General Procedure for the preparation of $9 \mathrm{a}, \mathrm{b}$ and 10

Method A. A mixture of each of (3a, b, 4) (0.1 mol) and $\mathrm{N}$-phenylmaleimide $(0.1 \mathrm{~mol})$ was heated at $200^{\circ} \mathrm{C}$ for $1 \mathrm{hr}$, left to cool and triturated with ethanol the solid product, so formed, was collected by filtration and crystallized from ethanol.

Method B. A mixture of each of (3a, b, 4) $(0.1$ mol) and $\mathrm{N}$-phenylmaleimide $(0.1 \mathrm{~mol})$ was placed in the microwave oven and irradiated at $450 \mathrm{~W}$ for 15-28 mins then left to cool to room temperature, and the solid was collected and crystallized from ethanol.

Compound 9a. was obtained as green crystals (method A: 52\%, method B, 15 min: 63\%) mp $185^{\circ} \mathrm{C}$. Analysis for $\mathrm{C}_{25} \mathrm{H}_{17} \mathrm{~N}_{3} \mathrm{O}_{5}(439.42)$ : Calcd: $\mathrm{C}$, $68.33 ; \mathrm{H}, 3.90 ; \mathrm{N}, 9.56$. Found: C, 68.10; H, 4.00; $\mathrm{N}, 9.650 \%$. IR $\left(\mathrm{v} / \mathrm{cm}^{-1}\right): 1728$ (CO cster) 1710 , $1695(\mathrm{CO})$ and 1642 (ring CO), 'H NMR ( $\delta \mathrm{ppm})$ : $1.43\left(\mathrm{t}, 3 \mathrm{H}, \mathrm{CH}_{3}\right), 4.2\left(\mathrm{q}, 2 \mathrm{H}, \mathrm{CH}_{2}\right), 7.16-7.76(\mathrm{~m}$, $10 \mathrm{H}, \mathrm{Ar}-\mathrm{H}) ; 8.09$ (s, 1H, H-9); $9.01 \mathrm{ppm}(\mathrm{s}, 1 \mathrm{H}, \mathrm{H}-5)$ ${ }^{13} \mathrm{C} \mathrm{NMR}(\delta \mathrm{ppm}): 14.27\left(\mathrm{CH}_{3} \mathrm{CH}_{2}\right) ; 62.85\left(\mathrm{OCH}_{2}\right)$; $107.79,110.12,116.47,125.94,126.46,128.35$, $128.93,129.12,129.28,129.38,131.34,134.79$, $136.83,137.99,140.46,148.68,160.87$ (aromatic and hetcrocyclic carbon) and 162.91, 165.96, 168.17, 198.20 (4CO). ms: $\mathrm{M}^{+}(439)$.

Compound $9 \mathrm{~b}$. was obtained as dark yellow crystals (method A: 58\%, method B, 28 min: 67\%) mp $158^{\circ} \mathrm{C}$. Analysis for $\mathrm{C}_{20} \mathrm{H}_{20} \mathrm{~N}_{4} \mathrm{O}_{6}$ (469.13): Caked: C, 66.52; H. 4.08; N. 8.95. Found: C, 66.45; H, 4.17 ; N. $9.82 \%$. IR $\left(\mathrm{v} / \mathrm{cm}^{-1}\right): 1738$ (CO ester) 1695 (CO) and 1642 (ring CO), 'H NMR ( $\delta \mathrm{ppm}): 1.43$ (t, $\left.3 \mathrm{H}, \mathrm{CH}_{4}\right): 3.67\left(\mathrm{~s}, 3 \mathrm{H}, \mathrm{OCH}_{3}\right) ; 4.2\left(\mathrm{q}, 2 \mathrm{H}, \mathrm{CH}_{2}\right)$; $7.02-7.52(\mathrm{~m}, 9 \mathrm{H}, \mathrm{Ar}-\mathrm{H}) ; 8.08(\mathrm{~s}, 1 \mathrm{H}, \mathrm{H}-9) ; 9.03$ ppm (s, 1H, H-5); ms: $\mathrm{M}^{+}$(469).

Compound 10. was obtained as oily crystals (method A: $72 \%$, method B, 28 min: $88 \%$ ) mp $165^{\circ} \mathrm{C}$. Analysis for $\mathrm{C}_{21} \mathrm{H}_{21} \mathrm{NO}_{4}$ (341.07): Calcd: C, $73.90 ; \mathrm{H}, 3.25 ; \mathrm{N}, 4.10$. Found: C, 73.87; H, 3.31; 
$\mathrm{N}, 4.13 \%$. IR (v/cm-1): 1710 (ring CO) $1695 \mathrm{~cm}^{-1}$ (amide CO), ${ }^{1} \mathrm{H}$ NMR $(\delta \mathrm{ppm})$ : 7.2-7.68 (m, 9H, Ar-H), 7.99 (s, 1H, H-7), 8.50 ppm (s, 1H, H-11). ms: $\mathrm{M}^{+}$(341).

Triethyl 5-amino-3, 4-dihydro-4-0xo-3-phenylphtha lazine-1,6,7-tricarboxylate 11

A mixture of $3 \mathbf{a}(0.1 \mathrm{~mol})$ and diethyl fumarate $(0.1 \mathrm{~mol})$ was placed in the microwave oven and irradiated at $450 \mathrm{~W}$ for $2 \mathrm{~min}$, then left to cool to room temperature, and the solid was collected and crystallized from ethanol.

Compound 11. was obtained as dark red crystals $\left(52 \%\right.$ ), mp $228^{\circ} \mathrm{C}$. Analysis for $\mathrm{C}_{23} \mathrm{H}_{23} \mathrm{~N}_{2} \mathrm{O}$, (453.44): Calcd: C, 60.92; H, 5.11; N, 9.27. Found: C, $60.89 ; \mathrm{H}, 5.14 ; \mathrm{N}, 9.29 \%$. IR $\left(\mathrm{v} / \mathrm{cm}^{-1}\right): 3505$ and $3320\left(\mathrm{NH}_{2}\right), 1745,1714,1710$ (ester $\mathrm{CO}$ ) and 1673 (ring CO), ${ }^{1} \mathrm{H}$ NMR (DMSO-d 6 $\delta$ ppm): 1.19 $1.44\left(\mathrm{~m}, 9 \mathrm{H}, 3 \mathrm{CH}_{3}\right) ; 3.44$ (s, $2 \mathrm{H}, \mathrm{NH}_{2}$ ); 4.20-4.42 (m, $\left.6 \mathrm{H}, 3 \mathrm{CH}_{2}\right) ; 7.25-7.60(\mathrm{~m}, 5 \mathrm{H}, \mathrm{Ar}-\mathrm{H}) ; 8.15 \mathrm{ppm}$ (s, 1H, H-8). ms: $\left(\mathrm{M}^{+}+1\right)(454)$.

2, 5-Diphenyltetrahydrocyclobuta[1,5-c;3,4-c'] dipyrrole-1,3,4,6-tetraone 24 and 2,5,8-Triphenylhexahydro-2,5,8-triazatrindene-1, 3, 4, 6, 7, 9. hexanone 25

Method A. $N$-phenylmaleimide was heated at $200^{\circ} \mathrm{C}$ for $1 \mathrm{hr}$, left to cool and triturated with ethanol the solid product, so formed, was collected by filtration and crystallized from ethanol.

Method B. N-phenylmaleimide $(0.1 \mathrm{~mol})$ was placed in the microwave oven and irradiated at 450 $W$ for 5 mins, then left to cool to room temperature, and the solid was collected and crystallized from ethanol.

Compound 24. was obtained as pink crystals (method A: 52\%, method B: 60\%) mp $252^{\circ} \mathrm{C}$. Analy- sis for $\mathrm{C}_{20} \mathrm{H}_{14} \mathrm{~N}_{2} \mathrm{O}_{4}$ (346.34): Calcd: $\mathrm{C}, 69.36 ; \mathrm{H}$, $4.07 ; \mathrm{N}, 8.09$. Found: $\mathrm{C}, 69.40 ; \mathrm{H}, 4.09 ; \mathrm{N}, 8.12 \%$. ${ }^{1} \mathrm{H}$ NMR $(\delta \mathrm{ppm}): 3.64-3.66(\mathrm{~m}, 4 \mathrm{H}, \mathrm{H}$-cyclobutane) and 6.83-7.64 ppm (m, 10H, Ar-H). ${ }^{13} \mathrm{C} \mathrm{NMR}$ ( $\delta$ ppm): 32.4 (cyclobutane carbon); 120.4, 124.1, $128.7,140.8$ (aromatic carbon) $175.20(\mathrm{CO}) . \mathrm{ms}$ : $\mathrm{M}^{+}$(346).

Compound 25. was obtained as beige crystals (method A: $50 \%$, method B: $64 \%$ ) mp $275^{\circ} \mathrm{C}$. Analysis for $\mathrm{C}_{30} \mathrm{H}_{21} \mathrm{~N}_{3} \mathrm{O}_{6}$ (519.50): Calcd: $\mathrm{C}, 69.36$; $\mathrm{H}, 4.07 ; \mathrm{N}, 8.09$. Found: $\mathrm{C}, 69.39 ; \mathrm{H}, 4.10 ; \mathrm{N}, 8.10 \%$. ${ }^{1} \mathrm{H}$ NMR $(\delta \mathrm{ppm}):$ 2.88-3.03 (m, 6H, H-cyclohexane) and 6.86-7.67 ppm (m, 15H, Ar-H). ${ }^{13} \mathrm{C}$ NMR ( $\delta$ ppm): 29.4 (cyclohexane carbon); 120.4, 124.1, 128.7, 140.8 (aromatic carbon) $173.80(\mathrm{CO}) \mathrm{ms}$ : $\mathrm{M}^{+}$(519).

\section{REFERENCES}

1. Abu-Shanab, F. A.; Wakefield, B. J.; Al-Omran, F.; Abdel Khalik, M. M. and Elnagdi, M. H. J. Chem. Res. (s) $1995,488(m) 2924$.

2. Al-Awadi, H.; Al-Omran, F.; Elnagdi, M. H.; Infantes, L. C.; Foces-foces, Jagerovic, N. and Elguero, J. Tetrahedron 1995, 51, 12745.

3. Nyiondi-Bonguen, E.; Sopbue Fondjo, E.; Fomum T.; and Dupp D. J. Chem. Soc. Perkin trans / 1994. 2191.

4. Erian, A. W.; Hafez, E. A. A.; Darwish E. S. and Elnagdi, M. H. Can. J. Chem, 1998, 76, 1038.

5. Al-Omran, F.; Al-Awadi, N.; Elassar, A. A. and ElKhair, A. A. J. Chem. Res. $1996(\mathrm{~s}), 20(\mathrm{~m}), 237$.

6. Caddick, S. Tetrahedron 1995, 51, 10403.

7. Dela Hoz, A; Diaz-Ortis, A.; Moreno A. and Langa, F. Eur. J. Org. Chem. 2000, 65, 3659.

8. Al-Zaydi, Kh. M.; Al-Shiekh, M. A. and Hafez, E. A. J. Chem. Res. 2000(s), 13(m), 0173 . 